\title{
Genomic profiling of type- 1 adult diabetic and aged normoglycemic mouse liver
}

\author{
Flávia G Ghiraldini ${ }^{1 *}$, André B Silveira ${ }^{2}$, Dirk A Kleinjan ${ }^{3}$, Nick Gilbert ${ }^{3}$ and Maria Luiza S Mello ${ }^{1}$
}

\begin{abstract}
Background: Hyperglycemia induces chromatin remodeling with consequences on differential gene expression in mouse hepatocytes, similar to what occurs during aging. The liver is the central organ for the regulation of glucose homeostasis and xenobiotic and lipid metabolism and is affected by insulin signaling. The precise transcriptional profiling of the type-1 diabetic liver and its comparison to aging have not been elucidated yet.

Methods: Here, we studied the differential genomic expression of mouse liver cells under adult hyperglycemic and aged normoglycemic conditions using expression arrays.

Results: Differential gene expression involved in an increase in glucose and impaired lipid metabolism were detected in the type-1 diabetic liver. In this regard, Ppargcla presents an increased expression and is a key gene that might be regulating both processes. The differential gene expression observed may also be associated with hepatic steatosis in diabetic mouse liver, as a secondary disease. Similarly, middle-aged mice presented differential expression of genes involved in glucose, lipid and xenobiotic metabolism. These genes could be associated with an increase in polyploidy, but the consequences of differential expression were not as drastic as those observed in diabetic animals.

Conclusions: Taken together, these findings provide new insights into gene expression profile changes in type-1 diabetic liver. Ppargcla was found to be the key-gene that increases glucose metabolism and impairs lipid metabolism impairment. The novel results reported here open new areas of investigation in diabetic research and facilitate the development of new strategies for gene therapy.
\end{abstract}

Keywords: Type-1 diabetes, Aging, Liver, NOD mouse, Gene expression

\section{Background}

Type-1 diabetes mellitus (T1DM) is an autoimmune disease caused by lymphocyte infiltration in the endocrine pancreas leading to destruction of $\beta$-cells and consequently, to hyperglycemia. Cardiovascular ailments, such as heart attack and atherosclerosis [1], increased prevalence of pancreas, colon and liver cancer [2]; retinopathies, nephropathies and skin conditions are examples of secondary complications caused by hyperglycemia that are accelerated when untreated. Aging shares a number of risk factors with diabetes, such as insulin resistance, higher cholesterol concentration and blood pressure, leading to the theory that diabetes promotes a premature and accelerated aging-like phenotype [3].

\footnotetext{
* Correspondence: flaviaghi@gmail.com

${ }^{1}$ Department of Structural and Functional Biology, Institute of Biology,

University of Campinas (Unicamp), 13083-862 Campinas, SP, Brazil

Full list of author information is available at the end of the article
}

Although T1DM starts in the pancreas, the liver is a central organ that regulates glucose homeostasis, xenobiotic metabolism and detoxification, steroid hormone biosynthesis and degradation and lipid metabolism [4].

Hyperglycemia promotes chromatin remodeling and increased polyploidy levels in hepatocytes from nonobese diabetic (NOD) mice; these alterations are similar, but not identical, to the changes observed in hepatocytes from old mice [5]. Recently, it has been observed that the alterations in chromatin organization that occur in hepatocytes from hyperglycemic NOD mice might be orchestrated by the $\mathrm{NAD}^{+}$-dependent histone deacetylases Sirt1 and Sirt6 [6]. Although these sirtuins were more abundant in the NOD hyperglycemic mice, their activity was unchanged because of limiting levels of $\mathrm{NAD}^{+}$, which could promote differences in gene expression patterns [6].

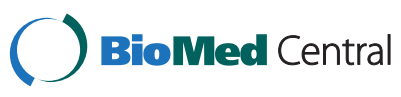


Genomic profiling studies have been conducted to investigate several organs in diabetic-related experimental conditions, including the type- 2 obese diabetic mouse model (fat pads, liver and skeletal muscle) [7], the type-1 diabetic NOD mouse model (pancreatic lymph nodes, spleen and peripheral blood cells) [8] and the streptozotocin-induced type- 2 diabetic mouse model (liver) $[9,10]$. However, there have been no studies investigating the gene expression profile of T1DM liver. In this regard, the NOD mouse model presents an advantage over drug-induced diabetes models because the disease in this mouse strain develops spontaneously; additionally, there is no controversy over whether differences in gene expression in the liver are caused by diabetes-induced agents. Moreover, given the similarities between diabetes and aging in mouse hepatocytes, such as increased polyploidy and chromatin remodeling, we also investigated the effect of hyperglycemia and aging on genomic expression patterns in mouse liver.

\section{Methods}

\section{Animals}

NOD/SHILTJ and Balb/c mice were maintained in an animal care facility on a 12-h light/dark cycle and received food and water ad libitum. The protocols involving animal care and use were conducted under the guidance issued by the Medical Research Council in Responsibility in the Use of Animals for Medical Research and Home Office Project License PPL 60/3785 (Edinburgh, UK) and by the Committee for Ethics in Animal Use of the University of Campinas, Brazil (registration no. 1608-1).

The glycemia levels of the animals were checked once a week up to $24 \mathrm{~h}$ before they were euthanized. Blood samples were obtained by caudal puncture and analyzed using the automatic Accu-Check Performa glucose meter (Roche Diagnostica do Brasil, Jaguare, Brazil). Glycemia levels within the $90-100 \mathrm{mg} / \mathrm{dL}$ (5.00-5.55 mmol/ L) range were considered normal, and glycemia levels over $500 \mathrm{mg} / \mathrm{dL}(27.5 \mathrm{mmol} / \mathrm{L})$ for three weeks were considered indicative of severe hyperglycemia. Four mouse groups were used in this study: 1) three normoglycemic Balb/c young-adults (8-weeks-old); 2) three normoglycemic Balb/c mice (47-weeks-old) 3) two severe hyperglycemic NOD adults and a technical replicate, and 4) normoglycemic NOD mice matched for age to the diabetic groups. The technical replicate was obtained mincing fragments of livers from two hyperglycemic NOD mice and processing them as a completely independent sample. For the microarray validation by qPCR five different animals of each group were used.

The animals were euthanized by cervical dislocation, and their livers were removed and frozen in liquid nitrogen for molecular assays.

\section{RNA preparation}

Total RNA was extracted from livers with Tri-Reagent (Sigma ${ }^{\oplus}$, St. Louis, EUA). Crude RNA samples were On-Column purified with the RNeasy mini kit (Qiagen ${ }^{\circ}$, Hilden, Germany) and analyzed with an Agilent 2100 BioAnalyzer (Agilent ${ }^{\circ}$, Santa Clara, USA) before being subjected to microarray studies. Only samples with an RNA Integrity Number (RIN) above 7 were further processed.

\section{cRNA synthesis, labeling and hybridization}

Total RNA (500 ng) was used to synthesize cRNA using the Illumina TotalPrep RNA Amplification kit (Illumina ${ }^{\oplus}$, San Diego, USA) according to the manufacturer's protocol. Hybridization, washing and scanning of Illumina Whole Genome Mouse WG-6v2 Gene Expression BeadChips were performed according to a standard protocol.

\section{Microarray analysis}

Raw signal intensity measurements, background correction and normalization of samples were processed with the Illumina GenomeStudio software. Unpaired Limma analyzes using the Limma package for the R-based Bioconductor software [11] were used to obtain differentially expressed probesets with $\mathrm{p}<0.05$ and fold-change $>2.0$. We obtained two separate differentially expressed gene lists corresponding to the comparison of Balb/c youngadults vs. aged Balb/c mice and the comparison of normoglycemic NOD mice vs. severe hyperglycemic NOD mice. The IPA software (Ingenuity Systems) was used to functionally interpret the lists of differentially expressed genes, and to analyze enrichment of gene ontology groups and regulation of molecular networks. The raw array data are available at the NCBI GEO database with the accession number GSE50613.

\section{Validation of microarray results by quantitative RT-PCR}

Validation of differential gene expression was performed for the Pck1, Igfbp1, Sirt1, Srebp1, Ppargc1a, Apoe and Foxo1 genes by quantitative RT-PCR using the protocol described above. Five animals were used for each experimental condition (Additional file 1). Relative expression was calculated using $\beta$-actin as the endogenous control.

\section{Results}

Of the 26,766 genes with established sequences available on the Illumina ${ }^{\circledR}$ microarray chip, 219 were found to be differentially expressed ( $F C>2.0 ; \mathrm{p}<0.05)$ under hyperglycemic conditions in comparison to normoglycemic conditions. Of these differentially expressed genes, 86 were up-regulated, and 133 genes were down-regulated. The full list of differentially expressed genes is presented in Additional file 2. The quantitative PCR using five biological replicates demonstrated the consistency of our global expression profiling results (Additional file 1). We 
observed that the canonical pathways enriched by hyperglycemia were mainly involved in carbohydrate and lipid metabolism (TR/RXR activation and PXR/RXR activation pathways), as well as in inflammatory signaling (crosstalk between dendritic cells and natural killers cells; primary immunodeficiency signaling; caveolarmediated endocytosis signaling; cytotoxic T-lymphocytemediated apoptosis of target cells) (Figure 1-A). Based on which genes were differentially expressed and their functions, a trend towards metabolic diseases such as hyperglycemia (diabetes mellitus), hepatic steatosis and hepatic cell death (Figure 1-B) could be identified.

From these data, Ppargcla (peroxisome proliferatoractivated receptor gamma coactivator 1 alpha) was found to be a key regulatory gene playing roles in two canonical pathways that were differentially expressed under hyperglycemic conditions (high p-values): the TR/RXR activation (thyroid hormone receptor) and PXR/RXR activation
(Pregnane X receptor) pathways (Figure 1-A). Ppargc1a transcribes the transcription factor PGC- $1 \alpha$ and is also involved in other pathways with lower p-values. In these pathways, Ppargc1a increased gluconeogenesis, carbohydrate and xenobiotic metabolism and repressed lipolysis (Figure 1-C). When the genes were analyzed from a functional perspective, a general decrease in the expression of genes related to lipid metabolism and molecular transport and an increase in the expression of genes involved with carbohydrate metabolism and detoxification were detected; these pathways form a "metabolic network" (Figure 2).

The middle-aged mice had 199 differentially expressed genes compared to the young-adult mice; of these, 122 were up-regulated, and 77 were down-regulated. The list of differentially expressed genes is presented in Additional file 3. The global expression profiling and qPCR results yielded similar trends, which can be
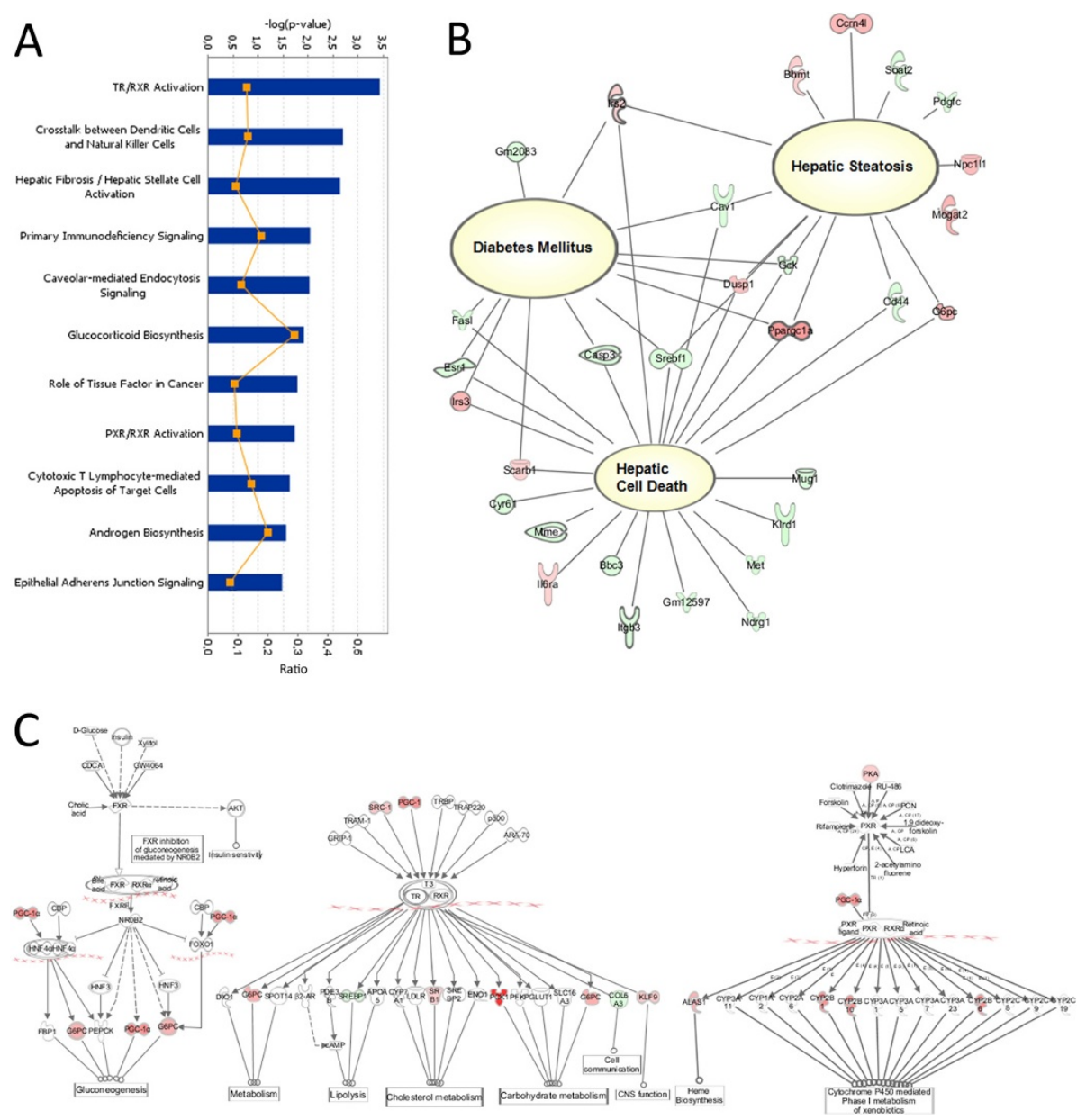

Figure 1 Diabetic NOD mouse panel. (A) The most activated canonical pathways in hyperglycemic NOD mice. -log(p-value), the probability that the association between the genes in the dataset and the canonical pathway is due to chance alone; ratio, the number of genes from the dataset that map to the pathway divided by the total number of genes that map to the canonical pathway. (B) Differentially expressed genes related to diseases found in the dataset. (C) Pregnane Receptor X and Thyroid Receptor canonical pathways. Genes in various shades of red indicate overexpression; genes in various shades of green indicate repression. 


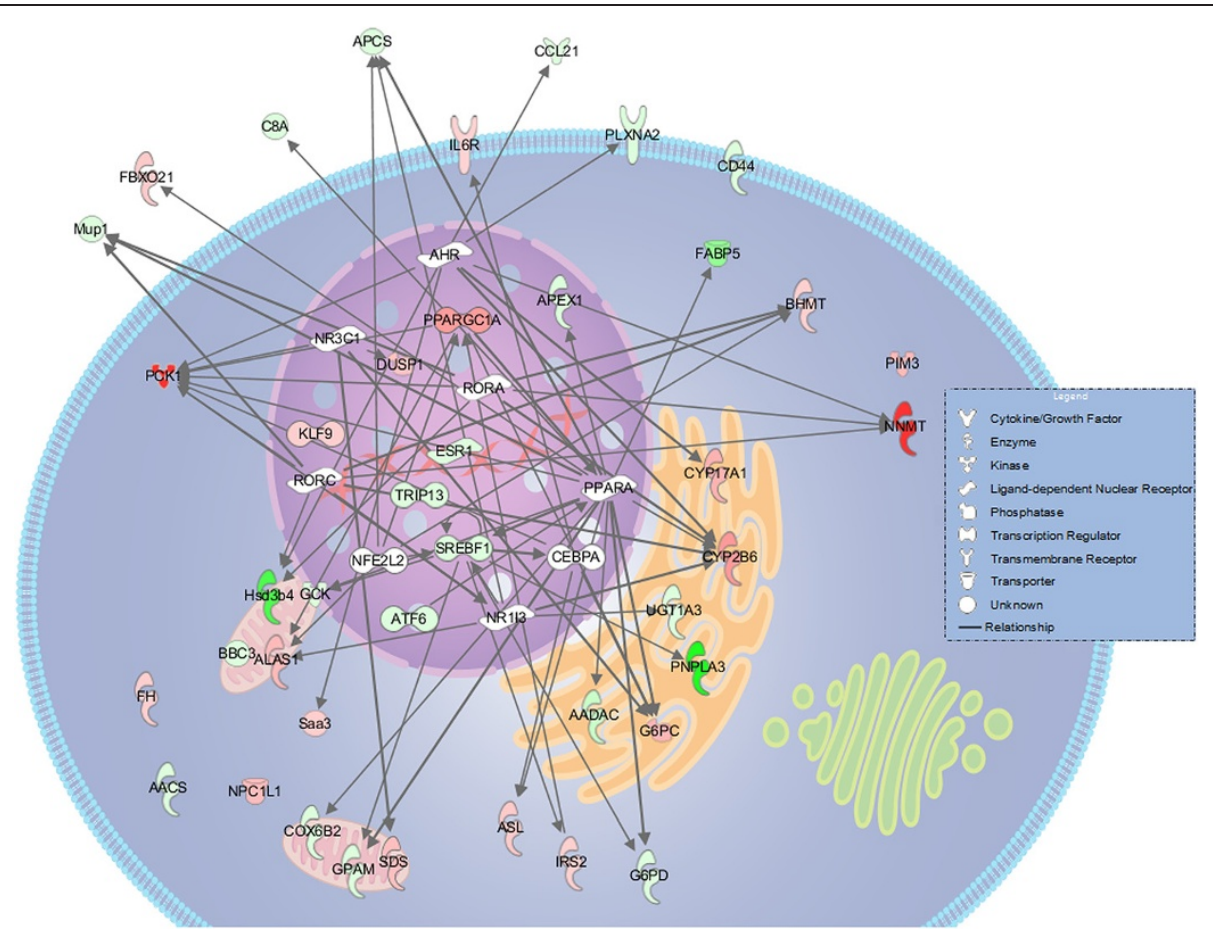

Figure 2 Metabolic network of diabetic NOD hepatocytes. Genes in shades of red indicate overexpression; genes in shades of green indicate repression; genes in white were not found to be differentially expressed in our dataset. Arrow, direct relationship.

observed in Additional file 1. Decreased lipid metabolism and increased hepatocyte proliferation were hallmarks of middle-aged mice (Figure 3-A). Among the most significant canonical pathways found differentially regulated in this experimental group, seven pathways indicated up-regulation of genes related to cytochrome P450 (Figure 3-B).

Considering all the genes that were differentially expressed in both experimental groups, a total of 23 genes were shared between adult diabetic and middleaged normoglycemic mice (Figure 3-C). Twelve of the shared genes were oppositely expressed in the two experimental groups, 11 were similarly expressed and were involved in circadian rhythm, lipid and carbohydrate metabolism (Figure 3-D). The 12 genes with inverted expression levels were related to several functions and could not be grouped into functional categories (Figure 3-D).

\section{Discussion}

Type-1 diabetes mellitus is an autoimmune disease that fundamentally produces an imbalance in carbohydrate and lipid metabolism. The genes that were differentially expressed in hyperglycemic animals played roles in the regulation of both metabolic processes and inflammatory signaling. Additionally, the transcription factor Ppargcla $(\mathrm{PGC}-1 \alpha)$ was a key upstream regulator. PGC-1 $\alpha$ acts with the histone deacetylase Sirt1 as a metabolic sensor in hepatocytes and increases the expression of genes involved in the gluconeogenesis pathway [12]. This study confirmed that PGC-1 $\alpha$ was up-regulated in hyperglycemic mice, a finding that has been demonstrated previously at the protein level [6], and highlighted Pck1 (phosphoenol pyruvate carboxykinase 1) and G6pc (glucose -6-phosphatase) as targets of PGC-1 $\alpha$. Pck1 and $G 6 p c$ are glycolytic genes active in the thyroid receptor pathway and induce gluconeogenesis [13].

PGC- $1 \alpha$ also plays a role in lipid metabolism. In the thyroid receptor canonical pathway, this protein induces the expression of Srb1 (Scavenger receptor B member 1 ), which increases the uptake of cholesterol esters from high-density lipoproteins (HDL) in the liver [14]. Furthermore, PGC- $1 \alpha$ decreases the expression of Srebp 1 (Sterol regulatory element-binding transcription factor 1), inhibiting fatty acid synthesis; this phenomenon has also been observed previously in Zucker diabetic fatty rats [15].

In untreated cases of T1DM and T2DM, fatty liver disease is a common secondary complication [16]. This is caused by an increased internalization of triglycerides in the liver, enhanced hepatic fat synthesis and decreased oxidation [17]. Indeed, we observed that the majority of the differentially expressed genes related to lipid metabolism in the diabetic mouse liver transcribe molecules that facilitate lipid uptake or regulate this process, such as Npc1l1 (Niemann-Pick C1-like 1), Srebp1, Bhmt (betaine-homocysteine S-methyltransferase) and Cav1 


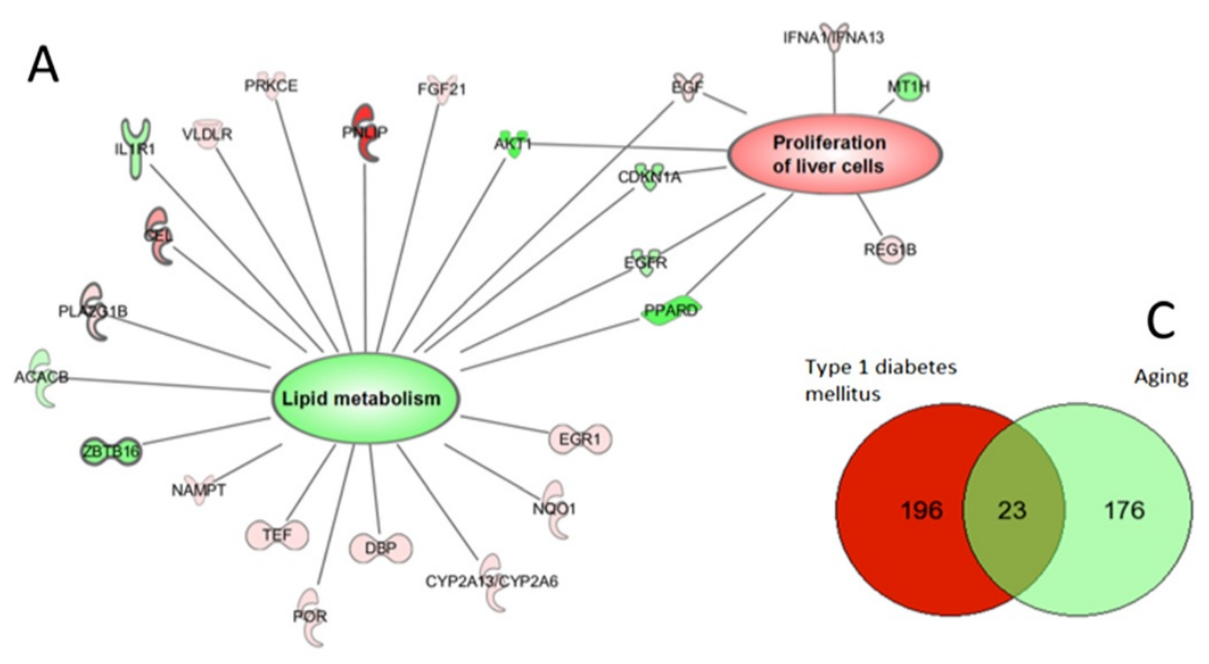

\section{B}

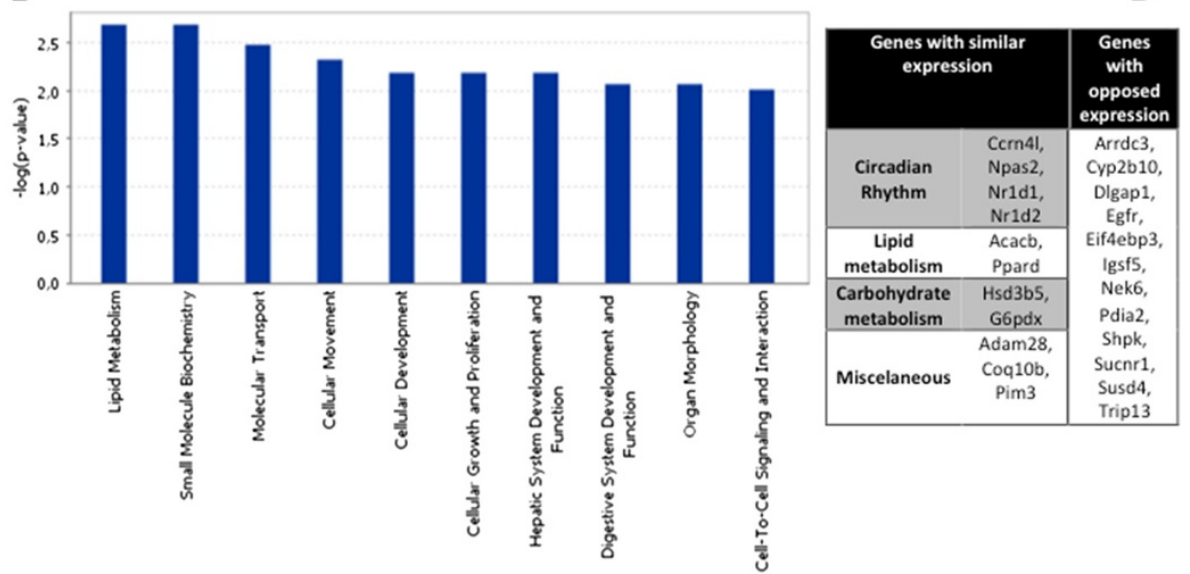

Figure 3 Middle-aged mice panel. (A) Differentially expressed genes related to altered biological functions in aging. Genes in shades of red indicate overexpression; genes in shades of green indicate repression. (B) The most frequently altered biological function in middle-aged mice hepatocytes. $-\log (\mathrm{p}$-value), the probability that the association between the genes in the dataset and the biological function is due to chance alone. (C) Venn diagram of genes differentially regulated in both diabetic and middle-aged mouse hepatocytes. (D) List of genes with inverted and similar expression between diabetic and middle-aged mice.

(Caveolin 1) [18-20]. The improper balance of genes related to lipid metabolism in diabetic NOD hepatocytes may promote the development of hepatic steatosis as a secondary complication, contributing to the aggravation of T1DM [21]. However, other genes, such as Srebp1 and Fabp5 (fatty acid binding protein 5) involved in the cholesterol biosynthesis and uptake, were downregulated. Therefore, although hepatic steatosis is suggested, no morphological or anatomic alterations could be identified in the samples analyzed. This might have occurred because alterations in the mRNA level could be still preliminary and might need longer period to fully develop into different phenotypes.

Similar to diabetes, middle-aged mice presented differentially expressed genes related to lipid metabolism. When the biological functions of these genes were analyzed, an overall decrease in genes involved in lipid metabolism was identified. The impaired lipid metabolism could be linked to the increased polyploidy previously observed in diabetic and aged mice [5], where a switch from oxidation of fatty acids to oxidation of glucose occurs [17]. This confirm the hypothesis that polyploidization is a response to high metabolic load and stress injury [22]. Oxidative DNA injury, similarly to that observed in T1DM [6] could impair proliferation, although promoting mitogenic stimulation following polyploidy [23]. The polyploidization process could enhance cell survival pathways and differential energy consumption in the liver preferring carbohydrates rather than fatty acids for ATP production [24].

Indeed, the misbalance on lipid and carbohydrate metabolism can be linked to increased production of 
reactive oxygen species (ROS), highly abundant in untreated cases of T1DM [25]. The ROS generated by excess glucose has been shown to increase DNA fragmentation and cell damage [6], which might contribute to differentially regulate genes involved in stress responses and growth arrest that leads to hepatic cell death. In fact, cytochrome P450 is involved in ROS formation [26]. Increased ROS has been considered as one of the hallmarks of aging and recently regarded as a stress-triggered survival signal $[27,28]$. As the organism gets older, ROS levels surpass the threshold that the cells could attempt to maintain for normal function [27]. In this study, two subunits of cytochrome P450 complex were found overexpressed in T1DM mice, which might contribute for the increase in DNA damage and be the effector of possible phenotypical changes [6]. The keygenes found here are responsible for the phase 1 of xenobiotic metabolism, when an initial modification and activation of typically lipophylic xenobiotics occur [29]. A counterbalance of four overexpressed and four downregulated subunits of the cytochrome $\mathrm{P} 450$ was observed in aged mouse liver; however there were also 16 overexpressed genes related to stress response. This response might also be a consequence of polyploidy because increased expression of genes involved in stress responses and chaperones occur as a physiological adaptation to aging [17]. Several differentially expressed genes in middle aged-mice were related to hepatocyte proliferation. Cdkn1a (p21), an inhibitor of cyclin-dependent kinases [30], Egf (Epidermal growth factor) [31] and Mt1h (metalloproteinase 1), which is a negative growth regulator [32], are genes involved in polyploidization and cell proliferation and were differentially expressed. During the polyploidization process the mouse hepatocytes may enter in mitosis with incomplete cytokinesis and present not only upregulated genes involved in cell proliferation but also with stress response $[24,33]$ similar to what was observed in this study. This observation indicates a possible trend toward polyploidization during the aging process, which is a well known phenomenon in mouse liver [5,34-36].

In diabetic NOD mice, livers also presented differentially expressed genes involved in immune signaling. The canonical pathways related to inflammation and immune response indicated a role for these genes in innate immunity, antigen presentation and immunodeficiency. The crosstalk between the dendritic cells and natural killer cells pathway has been related in other studies to be one of the responsible alterations found in NOD mice that could elicit T1DM [37,38]. In the liver of Type-1 diabetic NOD mouse, a small population of $\mathrm{B}_{220^{+}}$-precursors dendritic cells with strong immune regulatory properties is deleted, confirming that the liver may affect the development of the autoimmunity of diabetes in
NOD mice $[37,38]$. In general, most of the differentially expressed genes in these mice were down-regulated, including the bone marrow stromal cell antigen 2 (Bst2), Fas ligand (Faslg) and interferon regulatory factor 7 (Irf7). A differential expression of these genes has also been observed in Type-2 diabetic mouse hepatocytes [7]. It is known that with aging there is an accumulation of pro-inflammatory tissue damage and that senescent cells secrete pro-inflammatory cytokines, enhancing NF-kB signaling [28]. In this study, middle-aged mice presented 12 genes differentially expressed that were involved with inflammation, the most predominant class of which was represented by chemokines. This indicates that both type- 1 diabetic and middle-aged mice presented a misbalance in inflammatory response, although during diabetes it was due to the auto-immune characteristic of the disease.

\section{Conclusions}

Taken together, the present findings provide new insights into gene expression profile changes in type- 1 diabetic liver. These alterations have consequences for several biological processes and key genes, such as Ppargcla. This particular gene increases glucose metabolism and impairs lipid metabolism, possibly leading to hepatic steatosis. Moreover, the differential gene expression pattern observed in the middle-aged mouse liver could be associated with polyploidization processes and other physiological adaptations. The novel results reported here highlighted PGC- $1 \alpha$ as a key regulator in diabetes and should open new areas of investigation in diabetes research and even promote the development of strategies for gene therapy.

\section{Additional files}

Additional file 1: (A) List of the primers used for quantitative gene expression validation. (B) Gene expression validation.

Additional file 2: List of differentially expressed genes in diabetic NOD mouse hepatocytes.

Additional file 3: List of differentially expressed genes in middle-aged Balb/c mouse hepatocytes.

\section{Abbreviations}

Apoe: Apoliprotein E; Bhmt: Betaine-homocysteine S-methyltransferase; Cav1: Caveolin 1; Fabp5: Fatty acid binding protein 5; Foxo1: Forkhead box protein 1; G6PC: Glucose - 6 - phosphatase; HDL: High density lipoprotein; Igfbp1: Insulin growth factor-biding protein 1; NOD mouse: Non-obese diabetic mouse; Pck1: Phosphoenol pyruvate carboxykinase 1; PGC-1a/ Ppargcla: Peroxisome proliferator-activated receptor gamma coactivator 1-alpha; PXR: Pregnane X Receptor; ROS: Reactive oxygen species; Sirt1: Sirtuin 1; Sirt6: Sirtuin 6; Srb1: Scavenger receptor B member 1; Srebp1: Sterol regulatory element-binding transcriptor factor 1; T1DM: Type-1 diabetes mellitus; T2DM: Type-2 diabetes mellitus; TR: Thyroid hormone receptor.

\section{Competing interests}

The authors declare that they have no competing interests. 


\section{Authors' contributions}

FGG researched data and wrote the manuscript, ABS contributed to the statistical analysis and revised the manuscript, DAK provided the animals in Edinburgh and revised the manuscript, NG contributed to experiment design and revised the manuscript, MLSM contributed to discussion and revised the manuscript. All authors read and approved the final manuscript.

\section{Acknowledgments}

This work was supported by the São Paulo State Research Foundation (FAPESP-grants no. 2010/50015-6 and 2008/58067-5) and the Brazilian National Research and Development Council (CNPq - grants no. 471303/2009-7 and 301943/2009-5).

\section{Author details}

${ }^{1}$ Department of Structural and Functional Biology, Institute of Biology, University of Campinas (Unicamp), 13083-862 Campinas, SP, Brazil. ${ }^{2}$ Laboratory of Molecular Biology, Centro Infantil Boldrini, Campinas, SP, Brazil.

${ }^{3}$ Medical Research Council Human Genetics Unit, Institute of Genetics and Molecular Medicine, University of Edinburgh, Edinburgh, UK.

Received: 13 September 2013 Accepted: 25 February 2014 Published: 3 March 2014

\section{References}

1. Valentine N, Van de Laar FA, van Driel ML: Adenosine-diphosphate (ADP) receptor antagonists for the prevention of cardiovascular disease in type 2 diabetes mellitus. Cochrane Database Syst Rev 2012, 11:cd005449.

2. Onitilo AA, Engel JM, Glurich I, Stankowski RV, Williams GM, Doi SA: Diabetes and cancer: risk, survival and implications for screening. Cancer Causes Control 2012, 23:967-981.

3. Stout RW: Diabetes atherosclerosis and aging. Diabetes Care 1990, 13:20-23.

4. Cao SX, Dhahbi JM, Mote PL, Spindler SR: Genomic profiling of short- and long-term caloric restriction effects in the liver of aging mice. PNAS 2001, 98:10630-10635.

5. Ghiraldini FG, Silva IS, Mello MLS: Polyploidy and chromatin remodeling in hepatocytes from insulin-dependent diabetic and normoglycemic aged mice. Cytometry 2012, 81:755-764.

6. Ghiraldini FG, Crispim ACV, Mello MLS: Effects of hyperglycemia and aging on nuclear sirtuins and DNA damage of mouse hepatocytes. Mol Biol Cell 2013, 24:2467-2476

7. Lan H, Ravaglia ME, Stoehr JP, Nadler ST, Schueler KL, Zou F, Yandell BS, Attie AD: Gene expression profiles of nondiabetic and diabetic obese mice suggest a role of hepatic lipogenic capacity in diabetes susceptibility. Diabetes 2003, 52:688-700.

8. Kodama K, Butte AJ, Creusot RJ, Su L, Sheng D, Hartnett M, Iwai H, Soares $L R$, Fathman CG: Tissue- and age-specific changes in gene expression during disease induction and progression in NOD mice. Clin Immunol 2008, 129:195-201.

9. Dhahbi JM, Mote PL, Cao SX, Spindler S: Hepatic gene expression profiling of streptozotocin-induced diabetes. Diabetes Technol Ther 2003, 5:411-420.

10. Zhang F, Xu X, Zhang Y, Zhou B, He Z, Zhai Q: Gene expression profile analysis of type 2 diabetic mouse liver. Plos One 2013, 8:e57766.

11. Smyth GK: Limma: linear models for microarray data. In Bioinformatics and Computational Biology Solutions using $R$ and Bioconductor. 1st edition. Edited by Gentleman R, et al. New York: Springer New York; 2005:397-420.

12. Rodgers JT, Lerin C, Gerhart-Hines Z, Puigserver P: Metabolic adaptations through the PGC-1a and SIRT1 pathways. EBS Lett 2008, 582:46-53.

13. Singh BK, Sinha RA, Zhou J, Xie SY, You SH, Gauthier K, Yen PM: FoxO1 deacetylation regulates thyroid hormone-induced transcription of key hepatic gluconeogenesic genes. J Biol Chem 2013, 288:30365-30372.

14. Fluiter K, Berkel TJ: Scavenger receptor B1(SR-B1) substrates inhibit the selective uptake of high-density-lipoprotein cholesteryl esters by rat parenchymal liver cells. Biochem J 1997, 326:515-519.

15. Suh $Y H$, Kim Y, Bang JH, Choi KS, Lee JW, Kim WH, Oh TJ, An S, Jung MH: Analysis of gene expression profiles in insulin-sensitive tissues from pre-diabetic and diabetic Zucker diabetic fatty rats. J Mol Endocrinol 2005, 34:299-315.

16. Targher G, Bertolini L, Padovani R, Rodella S, Zoppini G, Pichiri I, Sorgato C, Zenari L, Bonora E: Prevalence of non-alcoholic fatty liver disease and its association with cardiovascular disease in patients with type- 1 diabetes J Hepatol 2010, 53:713-718.

17. Anatskaya OV, Vinogradov AE: Genome multiplication as adaptation to tissue survival: evidence from gene expression in mammalian heart and liver. Genomics 2007, 89:70-80.

18. Jia L, Betters JL, Yu L: Niemann-pick C1-like 1(NPC1L1) protein in intestina and hepatic cholesterol transport. Annu Rev Physiol 2011, 73:239-259.

19. Werstuck GH, Lentz SR, Daval S, Hossain GS, Sood SK, Shi YY, Zhou J, Maeda N, Krisans S, Malinow MR, Austin RC: Homocysteine-induced endoplasmatic reticulum stress causes dysregulation of the cholesterol and triglyceride biosynthetic pathways. J Clin Invest 2001, 107:1263-1273.

20. Fernandez-Rojo MA, Restall C, Ferguson C, Martel N, Martin S, Bosch M, Kassan A, Leong GM, Martin SD, McGee SL, Muscat GE, Anderson RL, Enrich C, Pol A, Parton RG: Caveolin-1 orchestrates the balance between glucose and lipid-dependent energy metabolism: implications for liver regeneration. Hepatology 2012, 55:1574-1584.

21. Williams KH, Shackel NA, Gorrel MD, McLenna SV, Twigg SM: Diabetes and nonalcoholic fatty liver disease: a pathogenic duo. Endocr Rev 2013, 34:84-129.

22. Pandit SK, Westendorp B, Bruin A: Physiological significance of polyploidization in mammalian cells. Trends Cell Biol 2013, 23:556-566.

23. Gorla GR, Malhi H, Gupta S: Polyploidy associated with oxidative injury attenuates proliferative potential of cells. J Cell Sci 2001, 114:2943-2951.

24. Anatskaya OV, Vinogradov AE: Somatic polyploidy promotes cell function under stress and energy depletion: evidence from tissue-specific mammal transcriptome. Funct Integr Genomics 2010, 10:433-446.

25. Berg JM, Tymoczko L, Stryer $L$ : The integration of metabolism. In Biochemistry. 5th edition. Edited by Berg JM, Tymoczko L, Stryer L. New York: WH Freeman; 2002.

26. Bondy SC, Naderi S: Contribution of hepatic cytochrome P450 systems to the generation of reactive oxygen species. Biochem Pharmacol 1994, 48:155-159.

27. Hekimi S, Lapointe J, Wen Y: Taking a "good" look at the free radicals in the aging process. Trends Cell Biol 2011, 21:569-576.

28. Lopez-Otin C, Blasco MA, Partridge L, Serrano M, Kroemer G: The hallmarks of aging. Cell 2013, 153:1194-1217.

29. Amador-Noguez D, Dean A, Huang W, Setchell K, Moore D, Darlington G: Alterations in xenobiotic metabolism in the long-lived little mice. Aging Cell 2007, 6:453-470.

30. Sheahan S, Bellamy COC, Treanor L, Harrison DJ, Prost S: Additive effect of p53, p21 and Rb deletion in triple knockout primary hepatocytes. Oncogene 2004, 23:1489-1497.

31. Mossin L, Blankson H, Huitfeldt H, Seglen PO: Ploidy-dependet growth and binucleation in cultured rat hepatocytes. Exp Cell Res 1994, 214:551-560.

32. Scibetta AG, Stantangelo S, Coleman J, Hall D, Chaplin T, Copier J, Catchpole S, Bruchell J, Taylor-Papadimitriou J: Functional analysis of the transcriptor repressor PLU-1/JARID1B. Mol Cell Biol 2007, 27:7220-7235.

33. Margall-Ducos G, Celton-Morizur S, Couton D, Bregerie O, Desdouets C: Liver tetraploidization is controlled by a new process of incomplete cytokinesis. J Cell Sci 2007, 120:3633-3639.

34. Gupta S: Hepatic polyploidy and liver growth control. Semin Cancer Biol 2000, 10:161-171.

35. Moraes AS, Guaraldo AM, Mello MLS: Chromatin supraorganization and extensibility in mouse hepatocytes with development and aging. Cytometry 2007, 71:28-37.

36. Celton-Morizur S, Desdouets C: Polyploidization of liver cells. Adv Exp Med Biol 2010, 676:123-135.

37. Liang X, Ma L, Thai NL, Fung JJ, Qian S, Lu L: The role of liver-derived regulatory dendritic cells in prevention of type-1 diabetes. Immunology 2006, 120:251-260.

38. Gao B, Jeong W, Tian Z: Liver: an organ with predominant innate immunity. Hepatology 2008, 47:729-736

doi:10.1186/1472-6823-14-19

Cite this article as: Ghiraldini et al: Genomic profiling of type- 1 adult diabetic and aged normoglycemic mouse liver. BMC Endocrine Disorders 2014 14:19 